\title{
Intranasal Stem Cell Secretome therapy to prevent COVID-19 complications
}

\author{
Avinash Sanap ${ }^{1}$, Ramesh Bhonde ${ }^{2}$, Avinash Kharat ${ }^{1}$, and Supriya Kheur ${ }^{1}$ \\ ${ }^{1}$ Regenerative Medicine Laboratory, Dr. D. Y. Dental College and Hospital, Pune, India \\ ${ }^{2}$ Dr D Y Patil Vidyapeeth University
}

June 16, 2020

\begin{abstract}
Acute respiratory distress syndrome (ARDS) is the key cause for the morbidity and mortality due to COVID-19 infections. Recent clinical evidences suggest chronic release of pro-inflammatory cytokines as a prominent etiological factor. Adult stem cells are known to secrete potent immunomodulatory and bioactive compounds. We propose a nebulizer assisted treatment of Mesenchymal Stem Cells (MSCs) Secretome as a therapy which will counterbalance the COVID-19 induced cytokine storm as well as promote the regeneration of alveolar tissue attributed to the intrinsic cytokines and growth factor present in the Secretome. Intranasal administration of Secretome will not only protect against the COVID-19 associated complications but also bring down the necessity of the ventilator reducing the burden on health care infrastructure.
\end{abstract}

\section{Hypothesis:}

From an outbreak to an epidemic, World Health Organization (WHO) declared the global spread of the COVID-19 as pandemic warranting co-ordinated efforts for research and development into Diagnostics, Novel Therapeutics and Repurposing of drugs for the control of COVID-19(WHO, 2020). Respiratory failure caused by acute respiratory distress syndrome (ARDS) is the leading cause of mortality. One of the root causes for the ARDS is the cytokine storm which is acquired by uncontrolled release of pro-inflammatory cytokine such as (IFN- $\alpha$, IFN- $\gamma$, IL-1 $\beta$, IL-6, IL-12, IL-18, IL-33, TNF- $\alpha$, TGF $\beta$, etc.) and chemokines (CCL2, CCL3, CCL5, CXCL8, CXCL9, CXCL10, etc.) by immune cells(Xiaowei Li, Manman Geng, Yizhao Peng, Liesu Meng, 2020).

Interestingly, recent report suggest that intravenous transplantation of MSCs decreased the over activated cytokine secreting immune cells such as CXCR3+CD4+ T cells, CXCR3+CD8+ T cells with concomitant decrease in C-reactive protein (CRP), Tumor Necrosis Factor- $\alpha$ (TNF- $\alpha$ ) and increase in the immunosuppressive cytokine IL-10(Leng et al. , 2020). To everyone's surprise, transplanted MSCs found to be resistant to the COVID-19 infection and exhibited higher expression of anti- inflammatory and trophic factors such as TGF- $\beta$, HGF, LIF, GAL, NOA1, FGF, VEGF, EGF, BDNF(Leng et al. , 2020).

In search of a definitive viable solution to the dreadful COVID-19 infection, we propose stem cell Secretome as a promising therapeutic option devoid of side effects. Secretome of MSCs which possesses potent immunomodulatory effects which can counterbalance the pro-inflammatory cytokines driven cytokine storm. As depicted in figure 1, conditioned medium of MSCs which can be easily obtained by in-vitro cell culture and can be locally administered through assisted nebulizer mask by inhalation as initially proposed by us for the treatment of chronic obstructive pulmonary disease (COPD)(Avinash Kharat, Vikrant Patil, Supriya Kheur, 2019). Local administration of Secretome gives upper hand over transplantation as it's a cell free therapy employing rapid action necessary as in the case of COVID-19 driven ARDS. Local and assisted administration of MSCs Secretome will not only help counter balance the cytokine storm driven by immunomodulatory 
properties but also promote the cell survival, wound healing and anginogenic potential of resident cells by its paracrine effect mediated by VEGF, EGF, HGF, FGF, CNTF, and various interleukins(Ullah, Subbarao and Rho, 2015). We strongly recommend MSCs Secretome nebulisation prior to the use of ventilator which would avoid further complications. Combinatorial strategy of antiviral drugs along with immunomodulatory, tissue protective and healing potential of Secretome may reduce the severity of the COVID-19. An urgent development is warranted on the MSCs Secretome based therapeutics specifically targeted towards ARDS to ensure the health and survival of human being.

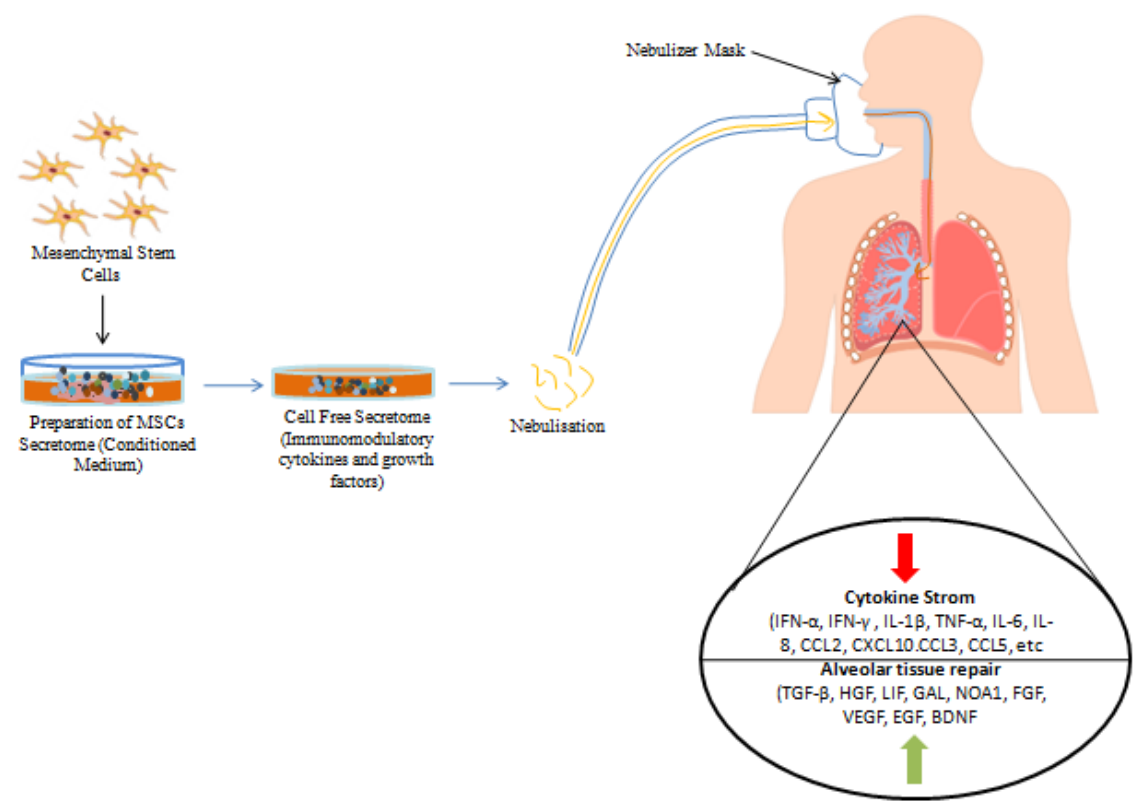

Figure 1 Intranasal Mesenchymal Stem Cell solution to COVID-19

Conflict of Interest: Authors declare no conflict of interest.

Funding: No funding received for this particular work.

\section{References:}

Avinash Kharat, Vikrant Patil, Supriya Kheur, R. B. (2019) 'Airway delivery of conditioned media from mesenchymal stem cells (MSC-CM) for COPD', Pulmonary Pharmacology 8 Therapeutics, https://do.

Leng, Z. et al. (2020) 'Transplantation of ACE2- mesenchymal stem cells improves the outcome of patients with COVID-19 pneumonia', ChinaXiv , 11(2), pp. 216-228.

Ullah, I., Subbarao, R. B. and Rho, G. J. (2015) 'Human mesenchymal stem cells - Current trends and future prospective', Bioscience Reports , 35. doi: 10.1042/BSR20150025.

WHO (2020) 'WHO COVID-19 Situation reports', (March). Available at: https://www.who.int/emergencies/diseases/novel-coronavirus-2019/situation-reports/.

Xiaowei Li, Manman Geng, Yizhao Peng, Liesu Meng, S. L. (2020) 'Molecular immune pathogenesis and diagnosis of COVID-19'. 